\title{
Influence of surface modification of fillers and polymer on flammability and tensile behaviour of polypropylene-composites
}

\author{
H. Demir, D. Balköse*, S. Ülkü \\ Izmir Institute of Technology, Faculty of Engineering, Department of Chemical Engineering, Gülbahce Campus, 35430 Urla Izmir, Turkey
}

Received 18 May 2005; received in revised form 28 June 2005; accepted 25 July 2005

Available online 30 September 2005

\begin{abstract}
An intumescent system consisting of ammonium polyphosphate (APP) as an acid source and blowing agent, pentaerythritol (PER) as a carbonific agent and natural zeolite (clinoptilolite, Gördes II) as a synergistic agent was used in this study to enhance flame retardancy of polypropylene (FR-PP). Zeolite was incorporated into flame retardant formulation at four different concentrations $(1,2,5$, and $10 \mathrm{wt} \%)$ to investigate synergism with the flame retardant materials. Filler content was fixed at $30 \mathrm{wt} \%$ of total amounts of flame retardant PP composites. Zeolite and APP were treated with two different coupling agents namely, 3-(trimethoxysilyl)-1-propanethiol and (3-aminopropyl)-triethoxysilane for investigation of the influence of surface treatments on mechanical properties and flame retardant performance of composites. Maleic anhydride grafted polypropylene (MAPP) was used for making polypropylene hydrophilic. Flammability of FR-PP composites was measured by the determination of limiting oxygen index (LOI). The LOI values reached to a maximum value of $41 \%$ for mercapto silane treated APP:PER (2:1) PP composite containing 5 wt $\%$ zeolite. The tensile strength of composites was increased by the addition of MAPP and elongation at break of composites was increased with silane treatments.
\end{abstract}

(c) 2005 Elsevier Ltd. All rights reserved.

Keywords: Natural zeolite; Mechanical properties; Flame retardant-polypropylene; Intumescent; Coupling agent; Polypropylene

\section{Introduction}

In recent years, polymeric composites were widely used in the production of new engineering materials. It is perceived as a reflection of technological development. Meanwhile the polymeric composites are promising, due to their economic versatile applicability and good mechanical properties. They are used in many applications, such as housing materials, transport and electrical engineering. Due to the increasing demand for polymers, polymeric materials bring new problems; the most important disadvantage of these materials concerns their low thermal resistance and fire behaviour [1-3]. For

\footnotetext{
* Corresponding author. Tel.: +90232 750 6270; fax: +90232 750 6196.

E-mail address: devrimbalkose@iyte.edu.tr (D. Balköse).
}

this reason, flame retardants are used. Flame retardants are defined as chemical compounds that modify pyrolysis reactions of polymers or oxidation reactions implied in the combustion by slowing down or by inhibiting them [4]. Many types of flame retardants are used in consumer products. They are mainly phosphorus, antimony, aluminium and boron-containing compounds, chlorides and bromides [5-7].

The flame retardant additives also affect mechanical properties of polymeric composites. The interface at the surface of particle inclusions plays a key role in the structure property relationship. For this purpose, many additives are employed for modifying surface of fillers and interface between fillers and polymeric matrix, i.e. coupling agents. The chemical structure of these additives allows combining the function of dispersing and coupling agents, so they are capable of bonding the filler and PP 
matrix by chemical bonds. Hence, mechanical properties are improved. Meanwhile flame retardancy could also be improved [8-13]. Polyboroxosiloxane, a reactive silicon oligomer, is used for modifying the surface of montmorillonite and ammonium polyphosphate (APP) particles. The limiting oxygen index (LOI) of polypropylene (PP) matrix composite increased from 31 to 37 by modification on surface of the particles [14]. Bertalan et al. modified the surface of APP with synergistic reactive surfactant that contains boron silicon segments. Increment of LOI of the composite was approximately $22 \%$ and relative elongation of composite increased by $50 \%$ [15].

Silane coupling agents have a general formula of $\mathrm{YSi}(\mathrm{OR})_{3}$, where R and Y stand for a hydrolysable group (methyl or ethyl) and a non-hydrolysable organofunctional group (amino, methacrylate, mercapto or vinyl groups), capable of interaction with fillers and polymers, respectively. Surface treatment of fillers with silane coupling agents was carried out through hydrolysis and condensation reactions as follows:

Hydrolysis reaction:

$\mathrm{YSi}(\mathrm{O}-\mathrm{R})_{3}+3 \mathrm{H}_{2} \mathrm{O} \rightarrow \mathrm{YSi}(\mathrm{OH})_{3}+3 \mathrm{ROH}$

Condensation reaction:

$\equiv \mathrm{Si}-\mathrm{OH}+\mathrm{YSi}(\mathrm{OH})_{3} \rightarrow \equiv \mathrm{Si}-\mathrm{O}-\mathrm{SiY}+\mathrm{H}_{2} \mathrm{O}$

Silanol groups formed as a result of a hydrolysis reaction react with hydroxyl groups found on filler surfaces to form siloxane bonds through a condensation reaction. As a result of these reactions, silane coupling agents modify the interface by forming a link between the components [16]. The polypropylene is grafted with maleic anhydride for increased polarity that leads to better adhesion with polar additives. The MAPP reveals two types of mechanisms. The first is the formation of ester bonds between the hydroxyl groups of additives and the anhydride carbonyl groups of MAPP. The second suggests the formation of an adhesive bridge between treated additives and polypropylene matrix, thereby increasing the interfacial adhesion between additives and polypropylene matrix [17].

Zeolite addition to intumescent formulations in the one hand makes the viscosity of molten polypropylene low by causing chain scission and thus expansion of foam to larger void volume becomes possible, and on the other hand increases the viscosity of the suspension as its volume fraction increases and prevents the expansion of the foam. Thus an optimum zeolite concentration should be present to get maximum foaming and highest LOI value.

The aim of this study was to investigate optimum zeolite concentration and the influence of surface modification on flame retardancy performance and mechanical properties of intumescent flame retardant polymeric matrix composites. Interphases of intumescent combinations from APP, PER, natural zeolite and polypropylene were improved by either silane treatments of APP and natural zeolite or by adding MAPP during preparation of composites. In this respect, surfaces of APP and natural zeolite particles were modified by coupling agents, 3-(trimethoxysilyl)-1-propanethiol (MS) and (3-aminopropyl)-triethoxysilane (AS) having thiol and amino groups to enhance the mechanical and fire retardant properties. It was thought that thiol or amino groups of coupling agents bonded to $\mathrm{OH}$ groups of inorganic fillers would react with the carboxylic acid groups formed on the surface of polypropylene during its melt processing in air and would form covalently bonded bridges between the filler and polypropylene. Maleic anhydride grafted polypropylene was also used for improving the interface of polypropylene and fillers. Flammability of samples was measured with limiting oxygen index (LOI), and thermal gravimetric analysis (TGA). Influence of surface treatments on tensile properties was investigated according to ASTM D-638 standards.

\section{Experimental}

\subsection{Materials}

Gördes zeolitic tuff having particle size below $50 \mu \mathrm{m}$ and supplied by Enli Madencilik Company was used in this study. The zeolite, which was characterised by a previous study of Top and Ülkü [18] was found to contain $80 \mathrm{wt} \%$ of clinoptilolite.

Exolit 422 ammonium polyphosphate (APP) $(n>$ 1000 ), having soluble fraction in water below $1 \%$, and average particle size of $15 \mu \mathrm{m}$, supplied by Clariant, pentaerythritol (PER), having particle size below $75 \mu \mathrm{m}$ supplied by MKS Marmara Kimya Sanayi A.S.., polypropylene (PP) MH 418, supplied by PETKIM A.Ş. and antioxidant, butylated hydroxy toluene, supplied by Sigma Co. were used in the experiments. The silane coupling agents were: 3-(trimethoxysilyl)-1-propanethiol (MS) having molecular formula of $\mathrm{C}_{6} \mathrm{H}_{16} \mathrm{O}_{3} \mathrm{SSi}$, supplied by Merck Co., and (3-aminopropyl)-triethoxysilane (AS) having molecular formula of $\mathrm{C}_{9} \mathrm{H}_{23} \mathrm{NO}_{3} \mathrm{Si}$, supplied by Fluka Co., MAPP, supplied by Clariant, were used for improving the surface of polypropylene.

\subsection{Surface treatments and compounding}

For surface treatments of inorganic fillers (clinoptilolite or APP), coupling agents (AS or MS), in an amount $1 \mathrm{wt} \%$ of fillers which is the optimum amount determined by Metin et al. [16], were added to $95 \mathrm{wt} \%$ ethanol solution and mixed for 15 min to allow silane hydrolysis. Then fillers (APP or Zeolite) were added to mixture in 1:1 
mass to volume ratio to solution and mixed for $45 \mathrm{~min}$ for condensation and chemical bonding of silanes and particles. Treated fillers were washed with ethanol to remove excess of coupling agents and dried in an oven at $70{ }^{\circ} \mathrm{C}$ overnight. The MAPP was added to replace $2.5 \mathrm{wt} \%$ of $\mathrm{PP}$ during mixing process by rheomixer.

Polypropylene matrix composites were prepared by blending of PP pellets, flame retardant materials (APP and PER), natural zeolite, clinoptilolite and antioxidant $(0.5 \%)$ using Haake Polydrive mixer. Concentration of fillers was fixed at $30 \%$ mass of total amounts of the composite. The optimum APP/PER ratio was determined as 2:1 in the previous work [19]. Zeolite was added with the mass fractions of 1,2,5 and 10\%. Clinoptilolite and APP (treated or untreated) were dried in an oven at $120{ }^{\circ} \mathrm{C}$ overnight. Samples were mixed at $60 \mathrm{rpm}$ screw speed at $190^{\circ} \mathrm{C}$ for $10 \mathrm{~min}$. At first, polypropylene was melted at $190{ }^{\circ} \mathrm{C}$ in plastograph for $2 \mathrm{~min}$ and then antioxidant $(0.5 \%$ in mass) was added to molten PP. Afterwards, clinoptilolite, APP (treated or untreated) and PER were added, respectively. Mixed materials were pressed into sheets having dimensions of $15 \times 15 \times$ $0.3 \mathrm{~cm}$ by Carver hot press at $190{ }^{\circ} \mathrm{C}$ and 100 bar. Composites were cut by bar shaped hollow die punch, with dimensions of $125 \times 6.5 \times 3 \mathrm{~mm}$ for LOI test.

\subsection{Test methods}

The limiting oxygen index (LOI) test apparatus for measuring the minimum oxygen concentration to support candle-like combustion of composites was constructed according to ASTM D-2863 [20]. The minimum concentration of oxygen in the mixture of oxygen and nitrogen flowing upward in a test column that supported combustion was measured under equilibrium conditions of candle-like burning. The gas flow rate in the column was adjusted by a Cole Parmer flowmeter (A-3227-30) to $4 \pm 1 \mathrm{~cm} / \mathrm{s}$.

TGA of composites was carried out using SETERAM Thermogravimetric Analyzer from room temperature to $800{ }^{\circ} \mathrm{C}$ at a heating rate of $10{ }^{\circ} \mathrm{C} / \mathrm{min}$. Dry air was used as a carrier gas with a constant flow rate during analysis.

Scanning electron microscopy (Philips XL-305 FEG SEM) was employed to examine the fracture surfaces of tensile tested specimens, and also to investigate the interface between filler and matrix and dispersion of filler in the matrix.

\section{Results and discussion}

\subsection{Flammability tests}

Table 1 summarizes the influence of coupling agent treatment on flame retardance performance of APP:PER
Table 1

LOI values of untreated and treated APP/PER (2:1) PP composites

\begin{tabular}{lllll}
\hline $\begin{array}{l}\text { Zeolite } \\
\text { concentration } \\
(\%)\end{array}$ & \multicolumn{4}{l}{ Limiting oxygen index (\%) } \\
\cline { 2 - 5 }$(\%)$ & Untreated & Amino silane & Mercapto & MAPP \\
\hline 0 & $38.0 \pm 1.8$ & $31.0 \pm 0.0$ & $29.4 \pm 0.0$ & $27.6 \pm 0.6$ \\
1 & $35.6 \pm 0.6$ & $37.0 \pm 0.0$ & $37.6 \pm 0.6$ & $29.0 \pm 0.7$ \\
2 & $35.6 \pm 0.6$ & $37.6 \pm 0.6$ & $38.0 \pm 0.0$ & $30.8 \pm 0.5$ \\
5 & $38.0 \pm 2.2$ & $40.6 \pm 0.9$ & $41.0 \pm 0.0$ & $26.4 \pm 0.6$ \\
10 & $29.0 \pm 1.0$ & $21.0 \pm 0.0$ & $28.0 \pm 0.0$ & $22.2 \pm 0.5$ \\
\hline
\end{tabular}

$(2: 1)+\mathrm{PP}+$ zeolite composites. The limiting oxygen index value decreased with surface treatment of fillers for composites without zeolite having only treated APP particles. The surface treatments caused antagonistic effect on flame retardancy of composites without zeolite formulation. Addition of the coupling agent treated zeolite to composites significantly affected the LOI values. At lower zeolite concentration of 1 and $2 \mathrm{wt} \%$, LOI values were improved about $4-8 \%$ with amino and mercapto silane treatments. At $5 \mathrm{wt} \%$ zeolite concentration, LOI value increased approximately $8 \%$ and reached to its maximum value of $41 \%$ with mercapto silane treatment. Higher zeolite loading $(10 \%)$ caused a decrease in the LOI value for both the untreated and treated composites. The limiting oxygen index test results indicate that MAPP addition have antagonistic effects on the LOI value of composites. Moreover, MAPP addition reduced the LOI value of composites lower than that without MAPP.

Consequently, while both mercapto and amino silane treatments increased LOI, MAPP addition caused a decrease in LOI. Furthermore, mercapto silane treatment enhanced the LOI values of composites more than amino silane treated composites. It is possible that AS and MS treatments on the surface of APP and zeolites have formed hydrogen bonding with pentaerythritol having reactive hydroxyl groups instead of non-polar polypropylene during processing. The treated APP and zeolite with pentaerythritol may have also formed a new structure that is much more resistant to heat and capable of forming char structure during thermal degradation.

\subsection{Thermal behaviour of composites}

Thermal behaviour of pure polypropylene, treated and untreated flame retardant-polypropylene (FR-PP) is shown in Figs. 1 and 2. Pure polypropylene began to degrade at $261{ }^{\circ} \mathrm{C}$ and $3.7 \%$ residue remained at $800{ }^{\circ} \mathrm{C}$ as shown in Fig. 1. Untreated FR-PP composites began to decompose at approximately $260{ }^{\circ} \mathrm{C}$ and lost most of its mass at $440{ }^{\circ} \mathrm{C}$. Beyond the temperature of $478{ }^{\circ} \mathrm{C}$, rate of mass loss slowed down and carbonaceous residue formed was about $24 \%$. Both TG curves of amino and mercapto silane treated FR-PP composites overlapped and also the same thermal behaviour was 


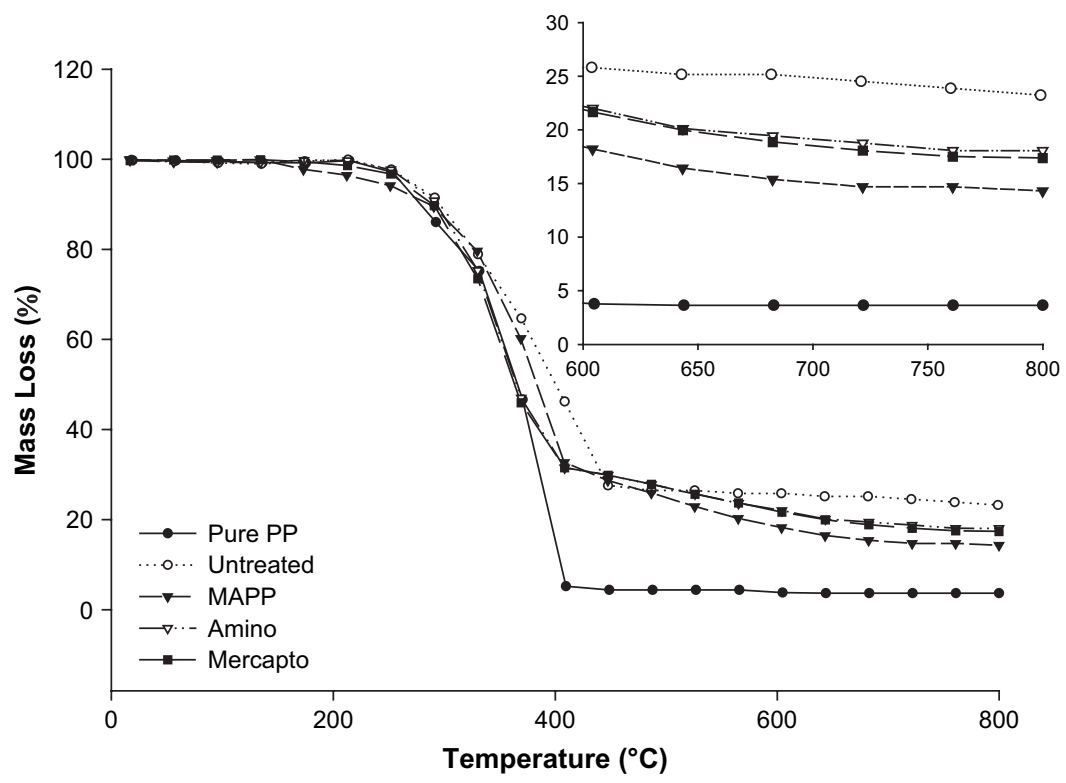

Fig. 1. TGA thermograms of untreated and treated FR-PP composites having 5\% zeolite and pure PP in dry air medium.

observed. They started to lose their mass at around $260{ }^{\circ} \mathrm{C}$ and the maximum mass loss was observed at $365^{\circ} \mathrm{C}$. Despite they lost big parts of their mass before the untreated FR-PP composites, the decomposition curves of both amino and mercapto silane treated FRPP composites shifted to higher temperatures than that of untreated ones, between 440 and $515^{\circ} \mathrm{C}$. At temperatures higher than $515{ }^{\circ} \mathrm{C}$, degradation starts to accelerate and carbonaceous residue obtained was approximately $18 \%$. It is possible that slow heating in TG made the silane treated FR-PP to decompose faster than untreated FR-PP in contrary of LOI results. It is well known that between 280 and $350{ }^{\circ} \mathrm{C}$ the development of intumescence occurs and between 350 and $430{ }^{\circ} \mathrm{C}$ the intumescence coating degrades. At higher temperatures, there are structural changes leading to formation of new carbonaceous species up to $430{ }^{\circ} \mathrm{C}$ [9]. As can be seen in Fig. 1, the new carbonaceous formation was propagated by silane coupling agents between 440 and $515^{\circ} \mathrm{C}$. Unusual results were observed for MAPP added FR-PP.

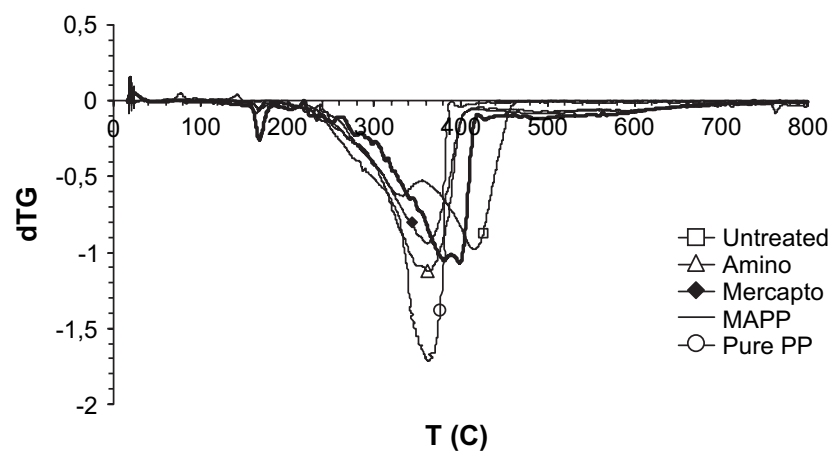

Fig. 2. DTG curves of untreated and treated FR-PP composites having $5 \%$ zeolite and pure PP in dry air medium.
The MAPP treated FR-PP composite degraded in two steps. Its degradation started at $170{ }^{\circ} \mathrm{C}$ and continued until $15 \%$ residue remained. It can be thought that degradation of composite was catalyzed by MAPP during preparation of composites. Generally, TG curves of MAPP added FR-PP composite confirmed LOI test results.

Fig. 2 illustrates DTG curves of treated and untreated FR-PP composites. Despite the fact that the pure PP and silane treated FR-PP lost big parts of their mass at the same temperature of $365^{\circ} \mathrm{C}$, the pure PP decomposed at higher rates than silane treated composites. Untreated FR-PP composite decomposed in two steps. First, intumescent coating degraded at around $325^{\circ} \mathrm{C}$ and remaining structure degraded at $420{ }^{\circ} \mathrm{C}$. In DTG curves of MAPP added FR-PP composite small peak was observed at $170{ }^{\circ} \mathrm{C}$. But the biggest mass loss was observed at $390{ }^{\circ} \mathrm{C}$ as second peak in Fig. 2 .

\subsection{Tensile behaviour of composites}

Influence of surface treatments of fillers with coupling agents on mechanical properties of composites are summarized in Tables 2-4. Tensile strength of silane treated and untreated composites did not show significant variation with the composition of composites and were close to each other. Surface treatments with coupling agents (AS and MS) did not modify the tensile strength properties of composites. The tensile strength of pure PP is around $33 \mathrm{MPa}$ which is higher than $30 \mathrm{wt} \%$ filler reinforced flame retardant PP composites. Since active sites of maleic anhydride grafted polypropylene improved adhesion with pentaerythritol and inorganic fillers (zeolite and APP), MAPP addition 
Table 2

Tensile strength (MPa) of untreated and modified flame retardant PP composites

\begin{tabular}{lllll}
\hline $\begin{array}{l}\text { Zeolite } \\
\text { loading (\%) }\end{array}$ & Untreated & AS & MS & MAPP \\
\hline 0 & $22.8 \pm 1.0$ & $23.6 \pm 0.3$ & $24.3 \pm 1.0$ & $25.6 \pm 1.3$ \\
1 & $19.2 \pm 0.8$ & $18.7 \pm 1.6$ & $17.7 \pm 0.5$ & $24.2 \pm 0.6$ \\
2 & $19.1 \pm 0.5$ & $18.5 \pm 0.4$ & $18.5 \pm 0.6$ & $24.6 \pm 1.5$ \\
5 & $18.6 \pm 1.1$ & $17.4 \pm 0.8$ & $17.7 \pm 0.8$ & $26.7 \pm 0.5$ \\
10 & $21.8 \pm 0.5$ & $22.5 \pm 0.5$ & $21.6 \pm 1.6$ & $27.9 \pm 0.2$ \\
\hline
\end{tabular}

enhanced the tensile strength of composites by $12-28 \%$ compared to untreated ones. Tensile strength of composites with MAPP increased with increasing zeolite content in the formulation as shown in Table 2. At $10 \%$ zeolite content, tensile strengths of treated and untreated composites were higher than that of lower zeolite content formulations.

Elongation at break values of untreated and treated flame retardant PP composites are revealed in Table 3 . Maximum elongations were 25.7 and $23.5 \%$ observed for $1 \%$ MS treated and $2 \%$ untreated zeolite-containing composites, respectively. Coupling agents enhanced the strain properties of FR-PP composites. The development of elongation properties can be a consequence of homogeneous structure and improved adhesion between the matrix and filler via the modified interface [15], although, increased elongation is not a common influence of coupling agents. In this study, the development of elongation may be caused by coupling agents which react with pentaerythritol and form strong structure between PER particles and inorganic fillers (APP and natural zeolite). Another reason can be the minimized formation of voids between fillers and polymeric matrix by coupling agents' treatments, thus deformations could not start so easily. Surface treatments with mercapto silane gave higher results than amino silane and MAPP addition for elongation properties of composites. Elongation of pure PP was $418 \%$. However, elongation properties of composite decreased to $32-45 \%$ with the increasing zeolite concentration up to $10 \%$.

In Table 4, Young's modulus of treated and untreated flame retardant PP composites is summarized. Young's modulus of pure PP is $1339 \mathrm{MPa}$ and $30 \mathrm{wt} \%$ filler reinforced FR-PP composites have Young's modulus

Table 3

Elongation at break (\%) of untreated and modified flame retardant PP composites

\begin{tabular}{lrlll}
\hline $\begin{array}{l}\text { Zeolite } \\
\text { loading (\%) }\end{array}$ & Untreated & AS & MS & MAPP \\
\hline 0 & $14.6 \pm 2.9$ & $17.0 \pm 3.5$ & $16.7 \pm 5.7$ & $17.5 \pm 2.8$ \\
1 & $16.8 \pm 4.1$ & $20.5 \pm 3.2$ & $25.7 \pm 5.9$ & $16.3 \pm 3.5$ \\
2 & $17.7 \pm 3.6$ & $19.2 \pm 5.8$ & $23.5 \pm 1.7$ & $18.4 \pm 1.5$ \\
5 & $16.9 \pm 1.6$ & $17.4 \pm 3.9$ & $20.8 \pm 2.9$ & $17.0 \pm 0.9$ \\
10 & $6.6 \pm 0.7$ & $11.4 \pm 4.8$ & $10.9 \pm 6.1$ & $10.9 \pm 6.1$ \\
\hline
\end{tabular}

Table 4

Young's modulus (MPa) of untreated and modified flame retardant PP composites

\begin{tabular}{lllll}
\hline $\begin{array}{l}\text { Zeolite } \\
\text { loading }(\%)\end{array}$ & Untreated & AS & MS & MAPP \\
\hline 0 & $1160 \pm 50$ & $1400 \pm 190$ & $1700 \pm 200$ & $1400 \pm 160$ \\
1 & $1900 \pm 150$ & $1700 \pm 230$ & $1600 \pm 90$ & $1900 \pm 200$ \\
2 & $1900 \pm 200$ & $1800 \pm 220$ & $1750 \pm 240$ & $2000 \pm 90$ \\
5 & $1900 \pm 150$ & $1900 \pm 140$ & $1450 \pm 150$ & $1900 \pm 100$ \\
10 & $1400 \pm 220$ & $1700 \pm 230$ & $1500 \pm 70$ & $1700 \pm 200$ \\
\hline
\end{tabular}

values between 1000 and $2000 \mathrm{MPa}$ varying with their composition and surface treatments. Zeolite loading, especially 5\% loading, increased Young's modulus. Surface treatments with coupling agents caused to decrease Young's modulus except for $10 \%$ zeolite loaded composites, since, coupling agents enhanced adhesion between inorganic fillers and pentaerythritol instead of polypropylene matrix. However, Young's modulus of MAPP added FR-PP composites was not significantly different than both silane treated and untreated composites.

Kerner's equation is also used to calculate the modulus of a composite having nearly spherical particles in the case of some adhesion between the phases as shown in Eq. (1). Kerner's model expresses the effect of filler concentration on Young's modulus of the polymer composite.

$\frac{E_{\mathrm{c}}}{E_{\mathrm{m}}}=1+\left[\frac{15\left(1-\nu_{\mathrm{p}}\right)}{8-10 \nu_{\mathrm{p}}}\right]\left[\frac{\Phi_{\mathrm{f}}}{1-\Phi_{\mathrm{f}}}\right]$

where, $E_{\mathrm{c}}$, elastic modulus of the composite; $E_{\mathrm{m}}$, Young modulus of the composite; $\nu_{\mathrm{p}}$ is the Poisson's ratio of the polymer, taken as 0.35 for isotactic polypropylene; $\Phi_{\mathrm{f}}$ is volume fraction of filler [16].

The comparison of theoretical and experimental Young's modulus of untreated FR-PP composites is shown in Fig. 3. Kerner's model predicted the experimental Young's modulus of FR-PP composites only at 1,2 , and $5 \%$ of zeolite loading for untreated, AS and MAPP added composites.

Consequently, it can be thought that the Kerner's model proved complexity of interactions between polymeric matrix and fillers. Coupling agents could not modify adhesion between fillers and polymeric matrix. It is possible that there have been interaction between the active sites of pentaerythritol and active sites of coupling agents. Thus, mechanical properties of composites were not improved as high as expected.

\subsection{Fracture surface of composites}

Fig. $4 a-d$ shows fracture surface of untreated and treated APP:PER (2:1) 5\% zeolite PP composites. The 


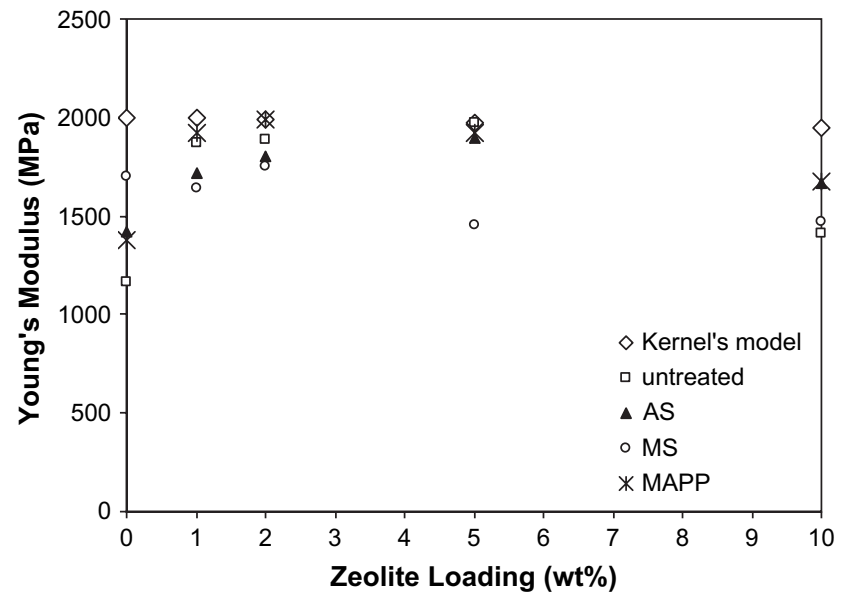

Fig. 3. Theoretical and experimental Young's modulus of untreated and modified flame retardant PP composites.

micrographs were taken in $200 \times$ magnification for representing general fracture surface of samples. In untreated sample, fillers did not adhere to polymeric matrix, however, ductile fracture of PP phase can be observed in Fig. 4a. Amino and mercapto silane surface treatments of APP and zeolite did not improve interface between fillers and polymeric matrix sufficiently as shown in Fig. $4 b$ and c. Particles did not adhere to the surface of polymeric matrix that cracks and voids can be observed around the particles clearly. The untreated, amino silane and mercapto silane treated composites showed ductile fracture as understood from fibrillated structure of matrix phase due to plastic deformation. MAPP added composite seemed to show brittle fracture as shown in Fig. 4d, since elongation in matrix phase was not observed.

\section{Conclusion}

The LOI values increased approximately $8 \%$ and reached to its maximum value of $41 \%$ for the MS treated composite containing $5 \mathrm{wt} \%$ zeolite. However, LOI values decreased for both the treated and untreated zeolite loaded at high level $(10 \%)$. The limiting oxygen index test results indicate that MAPP addition have antagonistic effects on the LOI value of composites. MAPP addition reduced the LOI to lower values than
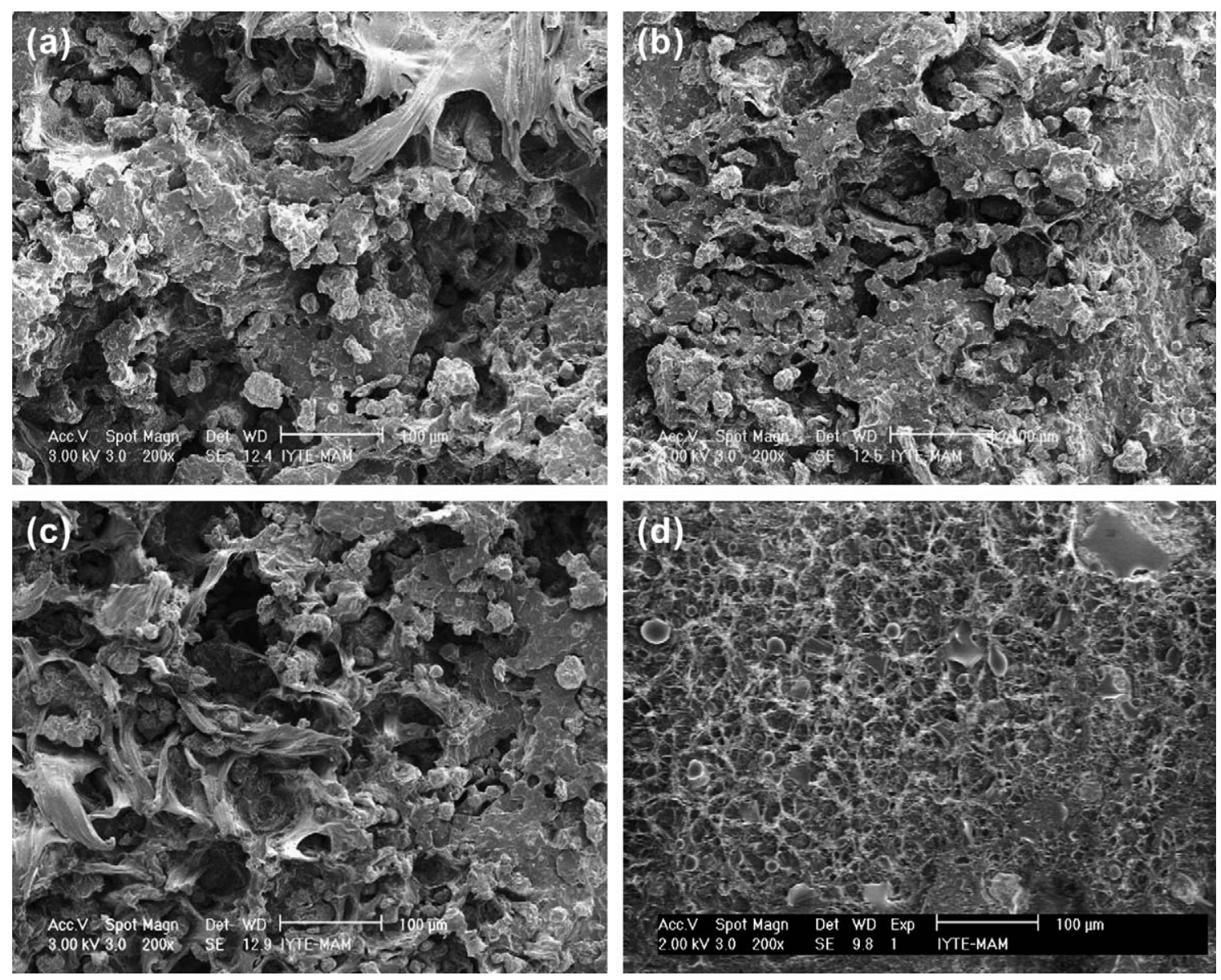

Fig. 4. SEM micrographs of fracture surface of tensile tested composites having 5\% zeolite untreated (a), amino silane (b), mercapto silane (c) and MAPP treated (d). 
that of untreated composites. The thermal degradation of both amino and mercapto silane treated FR-PP composites slowed down in the range of $440-515{ }^{\circ} \mathrm{C}$ compared to that of untreated composites. At temperatures higher than $515^{\circ} \mathrm{C}$, degradation began to accelerate and carbonaceous residue obtained was $18 \%$, which was lower than residue of untreated FR-PP composite. Thermal degradation of MAPP added FR-PP composite began at $170{ }^{\circ} \mathrm{C}$ and continued until $15 \%$ residue remained.

Surface treatments with coupling agents (AS and MS) did not enhance interface between polypropylene matrix and inorganic fillers, hence the tensile strength properties of composites did not improve. MAPP addition enhanced the tensile strength of composites by $12-28 \%$ compared to untreated ones. Coupling agents developed the strain properties of FR-PP composites. Young's modulus showed variation between 14007 and $1990 \mathrm{MPa}$ for $30 \mathrm{wt} \%$ filler reinforced FR-PP composites and it was $1300 \mathrm{MPa}$ for pure PP.

The composites contain pentaerythritol besides APP and zeolites. Pentaerythritol and polypropylene interphase should also be improved. Silane treatment for pentaerythritol was not possible since it was partially soluble in water. Consequently, coupling agents' treatments on surface of APP and zeolite could react with pentaerythritol during preparation of composites. The combination of treated fillers with pentaerythritol may have permitted the formation of a new structure that is much more resistant to heat. To explain the interaction between flame retardance performance and surface modifier, X-ray photoelectron spectroscopy (XPS) and NMR analysis should also be performed in further studies.

\section{References}

[1] Bourbigot S, Le Bras M, Breant P, Tremillon JM, Delobel R. Zeolite new synergistic agents for intumescent fire retardant thermoplastic formulations criteria for the choice of the zeolite. Fire and Materials 1996;20(3).

[2] Spirckel M, Regnier N, Mortaigne B, Youssef B, Bunel C. Thermal degradation and fire performance of new phosphonate polyurethanes. Polymer Degradation and Stability 2002;78:211-8.

[3] Bourbigot S, Le Bras M, Delobel R, Decressain R, Amourex JP. Synergistic effect of zeolite in an intumescent process study of the carbonaceous structure using solid-state NMR. Faraday Transactions 1996;92(1):149-58.
[4] Seymour RB. Additives for plastic. New York: Academic Press; 1978.

[5] Troitzsch J. International plastics flammability handbook. New York: Hanser Pub; 1990.

[6] Sain M, Park SH, Suhara F, Law S. Flame retardant and mechanical properties of natural fibre-PP composites containing magnesium hydroxide. Polymer Degradation and Stability 2004;83: $363-7$.

[7] Zhu S, Shi W. Thermal degradation of a new flame retardant phosphate methacrylate polymer. Polymer Degradation and Stability 2003;80:217-22.

[8] Ravadits I, Toth A, Marosi G, Marton A, Szep A. Organosilicon surface layer on polyolefins to achieve improved flame retardancy through an oxygen barrier effect. Polymer Degradation and Stability 2001;74:419-22. Elsevier Science Ltd.

[9] Almeras X, Le Bras M, Hornsby P, Bourbigot S, Marosi Gy, Keszei S, et al. Effect of fillers on the fire retardancy of intumescent polypropylene compounds. Polymer Degradation and Stability 2003;82(2):317-23. Elsevier Science Ltd.

[10] Anna P, Marosi Gy, Bourbigot S, Le Bras M, Delobel R. Intumescent flame retardant system of modified rheology. Polymer Degradation and Stability 2002;77:243-7.

[11] Zang X, Guo F, Chen J, Wang G, Liu H. Investigation of interfacial modification for flame retardant ethylene vinyl acetate copolymer/alumina trihydrate nanocomposites. Polymer Degradation and Stability 2005;87:411-8.

[12] Marosi Gy, Tohl A, Bertalan Gy, Anna P, Maatoug MA, Ravadits I, et al. Modified interfaces in multicomponent polypropylene fibers. Composites Part A Applied Science and Manufacturing 1998;29A:1305-11.

[13] Li B, He J. Investigation of mechanical property, flame retardancy and thermal degradation of LLDPE-wood-fibre composites. Polymer Degradation and Stability 2004;83:241-6.

[14] Marosi G, Marton A, Szep A, Csontos I, Keszei S, Zimonyi E, et al. Fire retardancy effect of migration in propylene nanocomposites induced by modified interlayer. Polymer Degradation and Stability 2003;82:379-85.

[15] Bertalan Gy, Marosi Gy, Anna P, Ravadits I, Csontos I, Toth A. Role of interface modification in filled and flame-retarded polymer systems. Solid State Ionics 2001;141-142:211-5.

[16] Metin D, Tihminlioglu F, Balköse D, Ülkü S. The effect of interfacial interactions on the mechanical properties of polypropylene/ natural zeolite composites. Composites Part A Applied Science and Manufacturing 2004;35:23-32.

[17] Kazayawoko M, Balatinecz JJ, Matuana ML. Surface modification and adhesion mechanisms in wood-fiber polypropylene composites. Journal of Materials Science 1999;34:6189-99.

[18] Top A, Ülkü S. Silver zinc, and copper exchange in a Na-clinoptilolite and resulting effect on antibacterial activity. Applied Clay Science 2004;27:13-9.

[19] Demir H, Arkış E, Balköse D, Ülkü S. Synergistic effect of natural zeolites on flame retardant additives. Polymer Degradation and Stability 2005;89:478-83.

[20] ASTM D 2863-91. Standard test method of measuring the minimum oxygen concentration to support candle-like combustion of plastics (oxygen index); 1991; 08.04 . 